\title{
IMPROVING THE RELIABILITY OF CABLE LINES OPERATION IN HOT CLIMATES
}

\author{
Madrakhimov Daniyar Bakhtiyarovich ${ }^{1 *}$, Ivanova Vera Pavlovna ${ }^{l}$ and Tsypkina Victoria Vyacheslavovna ${ }^{1}$ \\ ${ }^{1}$ Tashkent State Technical University named after I. Karimov, Tashkent, Uzbekistan
}

\begin{abstract}
Reliability of cable lines in hot climate is determined by the climatic characteristics of cables and wires, which include: long-term and short-term heat resistance, cold resistance, moisture resistance, resistance to cyclic exposure to temperatures and solar radiation, ozone resistance, etc. This article considers the main impacts of environmental factors: high temperatures, solar radiation, which, as practice shows, lead to irreversible deterioration of the electrical and mechanical properties of cable products. The result of climatic impacts in the Central Asian region, in hot climate conditions, is the aging of both insulation and protective coverings, which leads to irreversible change in the mechanical and electrical properties of the used polymers due to the loss of elasticity of the extruded material and its subsequent cracking, turning into cracks. The assessment of the possibility of long-term operation of the used polymer was carried out according to the polyethylene oxidation period, which determines the time of natural preservation of various types of cables during the period of their operation. The research was carried out on samples of cables stored under a canopy in wooden boxes, protected from sunlight and precipitation, by measuring criterion parameters with strict compliance with the established norms. Thus, the proposed solution for increasing the reliability of cable lines consists of debugging the technological process of applying insulation and sheathing, in which the extrusion of the polymer mass is carried out by technique that minimizes the ingress of contamination. Review of the results showed that extrusion line improvement would provide possibility of increasing reliability in the operation of cable products under the impact of climatic factors of the Central Asian region due to the reduced aging of insulation.
\end{abstract}

Keywords: reliability, climatic impacts, hot climate conditions, aging of insulation, cable and wire products, cable line, extrusion, extruder, catcher, technological process.

\section{Introduction}

Reliable operation of cable lines in hot climate is usually determined by the climatic characteristics of cables and wires, which include: long-term and short-term heat resistance, cold resistance, moisture resistance, resistance to cyclic exposure to temperatures and solar radiation, ozone resistance, etc. This article considers influence of major environmental impacts, such as high temperatures and solar radiation, which have negative impact on the efficient operation of the cable line. As practice shows, they lead to irreversible deterioration of the electrical and mechanical properties of cable products. In addition, as a result of the climatic impact in the Central Asian region (in hot climate conditions), this is rapid aging of both insulation and protective shells, which leads to an irreversible change in the performance characteristics of used polymers: loss of elasticity of the extruded material and its subsequent cracking, which turns into cracks.

Review of the research results showed [16, 17, 18, 19] that the improvement of the insulation application technology and modernization of the extrusion line would provide possibility of increasing reliability in the cable products operation. At the same time, in hot climates, it is also possible to achieve a decrease in the aging rate of cable insulation by improving technology of its application.

Reliability of cable lines is determined by the climatic characteristics of cables and wires, which include long and short-term heat resistance, cold resistance, moisture resistance, resistance to cyclical effects of temperature and solar radiation, ozone resistance, etc.

There is another problem of high ambient temperatures influence on the smooth operation of electrical systems, namely the temperature of the earth surface during summer. Based on the research results of Navalikhin E.Yu. and Trufanov N.M., conducted at FSBEI of Higher Education "Perm National Research Polytechnic University", it was found that a decrease in soil temperature led to a decrease in temperature in the cable channel [1], and therefore increased conductivity of the conductive part of cable line.

However, in winter there is a need to increase the load on the cable, which is justified by research of climatic

\footnotetext{
*Corresponding author: daniyar_19@yahoo.com
} 
conditions impact on the temperature field in the channel and can be determined by the choice of the transmission power level. At the time of rising consumer demand in winter, it becomes possible to increase the capacity of cable lines [1]. Depending on the temperature conditions (surface temperature range), power, transmitted through cable lines, is optimized by choosing the operating current. At the same time, experimental data on various temperature conditions of the surrounding soil (Table 1) allow us to establish recommended maximum permissible current loads, which depend on the temperature conditions of the surrounding soil and operational characteristics of the cable channel. The tabular data are given, taking into account the temperature of the XLPE insulation, which does not exceed the permissible temperature [1].

Table 1. Operation characteristics of the cable channel [1]

\begin{tabular}{|l|c|c|c|c|c|c|c|c|c|}
\hline \multicolumn{1}{|c|}{ Operation characteristics } & \multicolumn{7}{c|}{ Ambient ground temperature, ${ }^{\mathbf{0}} \mathbf{~ C}$} \\
\cline { 2 - 11 } & $\mathbf{- 2 0}$ & $\mathbf{- 1 5}$ & $\mathbf{- 1 0}$ & $\mathbf{- 5}$ & $\mathbf{0}$ & $\mathbf{+ 5}$ & $\mathbf{+ 1 0}$ & $\mathbf{+ 1 5}$ & $\mathbf{+ 2 0}$ \\
\hline $\begin{array}{l}\text { Maximum temperature in the channel, } \\
{ }^{0} \mathrm{C}\end{array}$ & 52 & 58 & 62 & 66 & 72,2 & 75,8 & 80 & 85 & 90 \\
\hline Operating current of cable lines, A & 115,7 & 115,7 & 115,7 & 115,7 & 115,7 & 115,7 & 115,7 & 115,7 & 115,7 \\
\hline $\begin{array}{l}\text { Permissible operating current } \\
\text { (temperature in the channel, 90 }{ }^{0} \mathrm{C}\end{array}$ & 140,7 & 136,6 & 133,3 & 130,4 & 127,7 & 125,3 & 122,8 & 119,5 & 115,7 \\
\hline Increased loading capacity, \% & 21,7 & 18,1 & 15,3 & 12,8 & 10,3 & 8,3 & 6,1 & 3,3 & 0 \\
\hline Total transmitted power, MVA & 62,9 & 61,1 & 59,6 & 58,2 & 57,07 & 55,94 & 54,89 & 53,39 & 51,72 \\
\hline
\end{tabular}

Studies of Russian specialists show that physical wear of cable lines is $70-80 \%$ with the value of their specific damage from 4.5 to 12 cases per $100 \mathrm{~km}$ per year, which is a very high rate of damage to cable lines [2], an example is the published information of JSC Russian Grids of analysis of the cable lines state $(0,4 \div 110(220) \mathrm{kV})$, which gives a general picture of damage [2], where:

- defects in laying of cable lines - 20\%;

- natural aging of power cables - $31 \%$;

- mechanical damage - 30\%;

- Factory defects - 10\%;

- Corrosion - 9\%.

Presented results show that the parameter "natural aging" takes the second position in the degree of damage to the cable and has the highest value in comparison with other parameters. One of the reasons for the manifestation of this negative factor is the excessive temperature and heating of the cable, which cause an imbalance between the heat, released inside the entire volume of the insulation, and the heat, removed to the environment, as well as possible thermal destruction of the insulation (dielectric), which entails an increase in the temperature of the dielectric, leading to its breakdown.

When laying cables in tunnels and channels, specialists determine the total heating of the ambient air during the calendar year and daily [3]. Change in the temperature of the ground layer around the cable line causes the cable temperature increase, leading to the damage to the entire line.

It has been established that daily changes depend on the layer thickness, which are considered depending on the annual temperature fluctuations at the same depth. Figure 1 shows the annual graphs of soil temperature changes, where the thermal parameters for the construction of these graphs are taken as averages from the relevant literature [17]. It is also taken into account that, depending on the season, temperature distribution changes at different depths throughout the year.

Based on the conducted measurements of soil temperature, based on annual temperature fluctuations and literature data, the dependence of changes in daily soil temperatures was built, taking into account seasonality of its distribution and climatic features in comparison between the Russian region and Uzbekistan. (fig. 1).

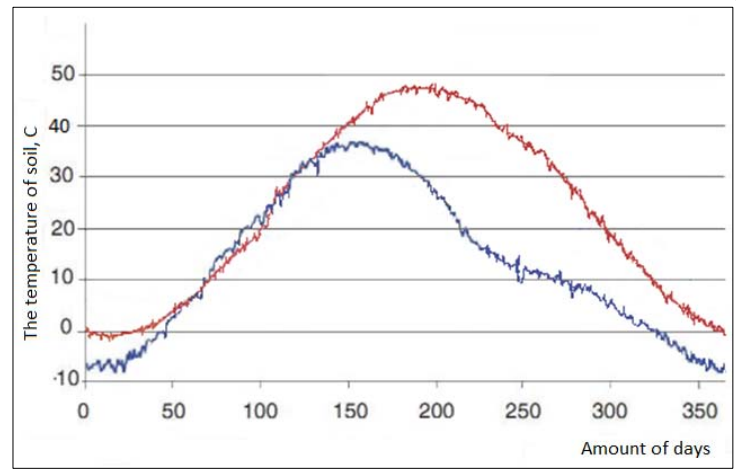

Fig.1. - Change in soil temperature for the Russian region and Uzbekistan throughout the year at average daily ambient temperatures.

In order to establish the terms of natural preservation of various types of cables during the period of their operation in hot season, studies were carried out at the Department of Electrical Machines of the Tashkent State
Technical University to assess the possibility of long-term operation of polymer, being used, according to the following parameters: insulation resistivity, dielectric 
constant, dielectric loss tangent and dielectric strength, change in leakage current $[6,7]$.

Cable samples in summer when exposed to high (daytime) temperatures, stored under a canopy in wooden boxes, protected from direct sunlight and atmospheric

Table 2. Electrical insulation properties of polymeric materials

\begin{tabular}{|c|c|c|c|c|}
\hline Material & $\begin{array}{l}\text { Specific volume electrical } \\
\text { resistance } \mathrm{Ohm}{ }^{*} \mathrm{~cm}\end{array}$ & $\begin{array}{c}\text { Electrical } \\
\text { strength, } \mathrm{kV} / \mathrm{mm}\end{array}$ & $\begin{array}{c}\text { Dielectric loss } \\
\text { tangent at } 1 \mathrm{KHz}\end{array}$ & $\begin{array}{c}\text { Dielectric } \\
\text { permeability }\end{array}$ \\
\hline PVC compounds & $10^{11} \div 10^{14}$ & $14 \div 20$ & $(50 \div 90)^{*} 10^{-3}$ & $3 \div 10$ \\
\hline Polyethylene & $10^{15 \div 10^{17}}$ & $18 \div 30$ & $(0,1 \div 0,3)^{*} 10^{-3}$ & 2,3 \\
\hline XLPE & More than $10^{15}$ & $25 \div 98$ & $0,3^{*} 10^{-3}$ & $2,3 \div 2,4$ \\
\hline $\begin{array}{l}\text { Polyethylene and its co- } \\
\text { polymers }\end{array}$ & $10^{14} \div 10^{15}$ & $25 \div 30$ & $(0,2 \div 0,3)^{*} 10^{-3}$ & $2,3 \div 2,4$ \\
\hline $\begin{array}{l}\text { Teflons: } \\
\text { PVDF(F-2) }\end{array}$ & More than $10^{14}$ & $10 \div 37$ & $(10 \div 20)^{*} 10^{-3}$ & $7,5 \div 13$ \\
\hline PTFE (F-4) & More than $10^{16}$ & $20 \div 30$ & $(0,2 \div 0,3)^{*} 10^{-3}$ & 2,0 \\
\hline
\end{tabular}

Termophysical properties of the environment around the cable have a negative impact on the temperature conditions of the cable product during the operation period, which means that these factors must be taken into account in calculating the load capacity of the cable product. Analysis of the data, given by Chirkin V.S., reflect the dependence of the thermophysical properties of conductive cores and metal screens on the design parameters of the conductor (bus duct), and, consequently, on the manufacturing technology [5]. precipitation, were studied by measuring criterion parameters with strict adherence to established operating standards (Table 2).

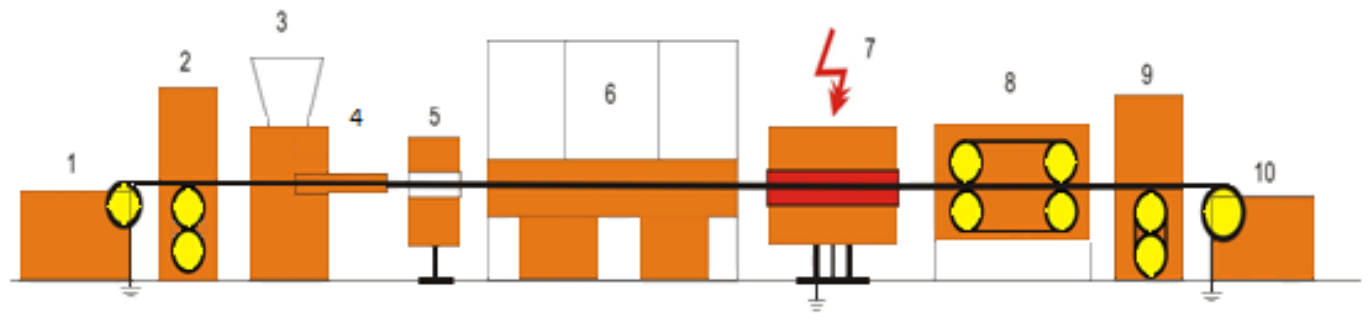

Fig. 2 Technological scheme of extrusion line

1-pay-off device; 2- compensator of the pay-off device; $\mathbf{3}$ - extruder;

4- extrusion head; 5- eccentricity and diameter meter; 6- cooling bath; 7 - SDIC; 8 -pull-off device; 9- compensator; 10- receiving device

Technological process of applying polymer insulation on the metal core (Fig. 2) is as follows: the metal core (copper, aluminum, non-ferrous metal alloys) from the pay-off drum (1) goes to the preheating device (2) (heating to $\mathrm{T}=90-100^{\circ} \mathrm{C}$ ) and then into the extruder head (4), in which the insulating layer is formed. Insulated core, going out from the working head of the extruder, cooled in the bath (6), enters the chamber for monitoring the continuity of the insulating layer (7) of SDIC (sound device of insulation continuity control). Checked wire in the control area (7) passes through the drum of the pulloff device (8) and enters the receiving device (10). Thickness of the applied insulation on the currentcarrying conductor and its eccentricity is regulated by the diaphragm device [8].

Modern extrusion lines have a very high degree of automation, combined with synchronization of operation of the main working nodes of the equipment. At the same time, the quality of applied insulation layer depends on [9]:
Technical solution, developed within the framework of ongoing research, is focused on increasing the reliability of cable lines. The essence of the development is to modernize technological process of applying insulation, in which extrusion of polymer mass is carried out according to the technique that minimizes the ingress of contaminants from the airspace of the production area of the workshop.
- maintaining and providing cleanliness in the material feed zone in the extruder feed area;

- keeping of technology tool in accordance with the established requirements;

- choice of temperature (right selection) in the work area depending on the parameters of the material;

- choosing and maintaining the temperature regime for cooling of the isolated blank.

Literature review showed $[16,17,18]$ that the improvement of the extrusion line, namely the polymer loading section, would provide the possibility of increasing the reliability of the cable product during the operation of cable products under the influence of climatic factors of the Central Asian region by reducing the aging index of insulation. The term "electrical aging of insulation" includes the gradual accumulation of micro cracks. The reason for the formation of these defects are electrical discharges, formed due to the presence of foreign inclusions in the insulation volume, which are influenced by the electromagnetic field, created by the conductive part of the cable. 
Contaminants and impurities in the cable insulation are formed as a result of mixing polymer beads (granules) and shop air in the extruder loading zone. At the same time, the extrusion section is not a "clean zone" of the production area of the workshop, where the air space is common for the entire workshop, which contains large amount of dust, generated during technological cycles. This is due to the close proximity on the production area of machines of various sections, including stranding (twisting) area, which ensures the presence of various fine, lightweight inclusions in a single airspace. Twisting of aluminum and copper wires into a core, of insulated electrical conductors, applying of armor and screens is carried out with the obligatory use of calibers that form and consolidate the twisted core (lay of armor, lay on twisting, screen), which means they mechanically act on metals and polymers by peeling off the top layer and forming those very lightweight and finely dispersed inclusions that constantly pollute the airspace of the workshop

However, when a layer of insulation is applied, in the case of detecting defects in the insulation at SDIC (Fig. 2 ), the line does not immediately stop, because there is a line speed lag. The location of the insulation defect is detected visually by the operator, which does not exclude the presence of human factor and error. Therefore, it is very important to ensure the cleanest possible supply of polymer materials to the loading zone of the extruder, minimizing the ingress of various kinds of lightweight and finely dispersed foreign particles into the polymer melt, which, mixing with the main insulating mass, form an insulation layer with defects and impurities along the current-carrying conductor.

Modern structures of extrusion lines allow fast loading of polymer due to the forced supply of material to the loading hopper, but at the same time, purification of air masses, coming from the workshop when loading granules, is not provided. Air, coming from the production hall to the extruder loading area, is not purified, because contains dust and various lightweight particles - the remnants of technological cycles, which are mixed with polymer granules during loading in the material molding zone (melt dosing zone) when the insulation layer of the conductive part of the cable is formed. (fig.3).

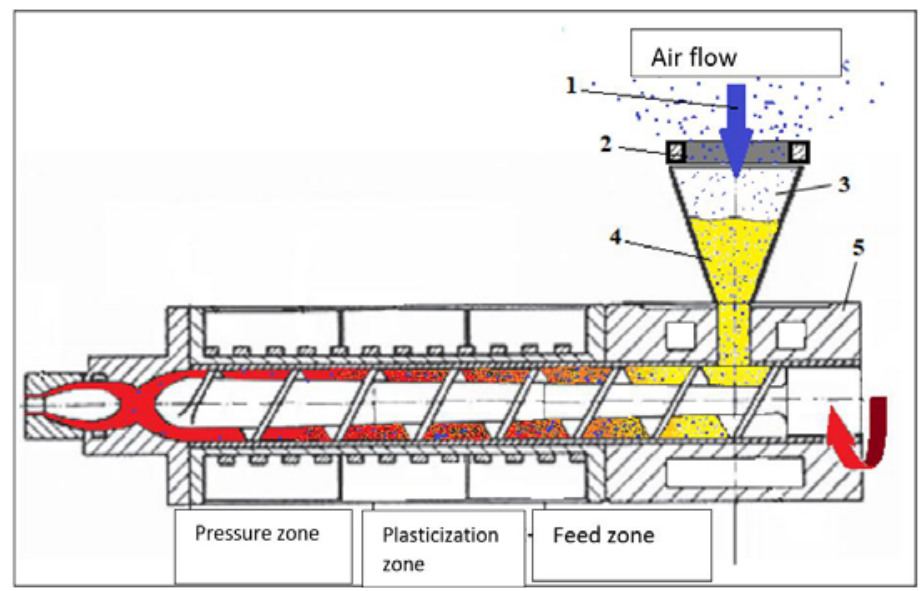

Fig. 3 - The scheme of the modernized extruder.

1- Air from the production area (uncleaned air flow); 2- catcher; 3- loading zone; 4- polymer granules; 5- extruder

To create conditions for minimizing the ingress of associated inclusions into the working area is one of the main tasks, the solution of which will increase the dielectric strength of the insulation and, as a result, increase the reliability of the cable as a whole.

The proposed technical solution will improve the reliability of cable lines by modernizing the process of applying insulation and sheathing, in which the extrusion of polymer mass is carried out according to the new technique, which ensures minimization of the ingress of contaminants and various kinds of inclusions into the extruded material.

Set goal is achieved by installing a catcher (2) in the loading zone of the extruder (Fig. 3), which purifies the air mass (1), accompanying the polymer granules (4). The catcher (4) is based on the method of air purification particles settling - from associated inclusions (fine, lightweight particles) by means of electrostatic force impact, which is also used in electric filters. The level of contamination of the dispersed medium is controlled by a laser dust sensor.

The proposed technical solution comes down to installing an electromagnetic coil in the loading area, the main functional load of which is to clean the air flow (in saturated state) by separating particles (contaminants) on the working surface of the catcher. Experimental tests made it possible to obtain the results of fractional efficiency depending on the amount of capturing (Table 3).

Table 3. Efficiency of the element depending on the diameter of the particles and the degree of capturing

\begin{tabular}{|c|c|c|c|c|}
\hline Particles diameter, $\boldsymbol{\mu m}$. & $\mathbf{1 0}$ & $\mathbf{2 0}$ & $\mathbf{3 0}$ \\
\hline Relative capturing & 0 & 3 & 17 & 30 \\
\cline { 2 - 5 } amount, \% & 4 & 20 & 70 & 92 \\
\cline { 2 - 5 } & 6 & 27 & 80 & 98 \\
\cline { 2 - 5 } & 8 & 40 & 85 & 99 \\
\hline
\end{tabular}


It is found experimentally (in laboratory conditions) that separation factor increases sharply with increase in the degree of capturing to $10 \%$ and increases slightly with further increase in collection efficiency. The improvement of the extrusion line made it possible to significantly increase the overall efficiency of the process equipment due to the forced cleaning of air flow. The optimum average flow rate in the installation is $7 \mathrm{~m} / \mathrm{s}$, while the dust concentrator collects the total amount of dust, the content of which is about $35 \%$.

Analysis of publications, devoted to the motion of gas mixtures $[10,11,12,13,14,15]$ on separation of single particles, laws of their motion, the peculiarities of the movement of the dispersed phase in chambers of various designs, sliding of the material relative to the flow of the carrier medium depending on Stokes, Froude, Reynolds numbers, made it possible to conclude that the calculation of the proposed system must be carried out according to the diameter and concentration of particles. In the production areas of the cable shop, gas mixture contains impurity particles with a size of 0.01-0.55 microns, while the true density indicator is $1750-2000 \mathrm{~kg} / \mathrm{m}^{3}$, and density value is $80-300 \mathrm{~kg} / \mathrm{m} 3$. Based on the foregoing, in the air flow of the shop that accompanies PVCcompound granules, content of fine foreign inclusions is $4-15 \%$, and accordingly the rest of the volume is the gas phase [16-19].

Thus, the operation of the dust collection unit allows the complex cleaning of the workshop air flow, both from metal inclusions and from dust, with obtaining the maximum effect in terms of: reducing the concentration of contaminants (lightweight, fine particles) when the air flow enters the feed zone of the extruder by $5-7 \%$; improving the quality and uniformity of the extruded insulating layer by $3-5 \%$; increasing the reliability of the cable product operation by increasing the dielectric strength of the insulation by $3-5 \%$.

\section{References}

1. Navalikhina E.Yu, Trufanova N.M.. Computer model of heat and mass transfer processes in the cable channel under various operating modes of cable lines // Science Journal « Fundamental research », 2014. № 9 (Part 5)- p. 988-992

2. Hoshimov, F.A., Bakhadirov, I.I., Erejepov, M., Djumamuratov, B. (2019) Development of method for normalizing electricity consumption E3S Web Conf 139 doi:10.1051/e3sconf/201913901074

3. Diagnostics // Publishing house «Open systems» [Electronic source] Link: http://www.osp.ru/lan/2003/12/138371/

4. On the use of XLPE insulated cables / G. Nevar // Cable news. - 2011. - №3.

5. Chirkin V.S. Thermophysical properties of materials. Handbook. M.: PHIZMATGIZ. 1959. - 356 p

6. High-density polyethylene // Chemical Industry Analytical Portal [Electronic source] Link: http://newchemistry.ru/material.php?id=3

7. The use of polymer materials in the cable industry / R. I. Ableyev // Polyurethane technology. - 2008. - №4
8. Manufacture of products from polymer materials "Ed.V.K. Kryzhanovsky, publishing house Profession, SPb. 2004.

9. Manufacturing technology // Estralin [Electronic source] Link: http://estralin.com/product/cable110/productiontechn ology/

10. Pirumov A.I. Aerodynamic foundations of inertial separation. M.:

1. Gosstroyizdat, 1961, $170 \mathrm{p}$.

11. Goldshtik M.A., Leontiev A.K., Paleev I.I. Movement of small particles in swirling flow // EPhJ. 1960, v.3, №2, p. 17 -24.

2. 12 Goldshtik M.A., Sorokin V.N. On the motion of a particle in the vortex chamber. //AMTPh. 1968, №6, p. $149-152$.

12. Uzhov V.N., Valdberg A.Yu, Myagkov B.I., Reshidov I.K. Cleaning of industrial gases from dust.- M.: Chemistry, 1981.-392 p.

13. Gupta A, Lilly D., Saired N. Swirling flows. - M.: World, 1987. -588p.

14. Strouss V. Industrial gas cleaning.: Translation from English. -M.: Chemistry, 1981, 616 p.

15. Tadmor Z., Gogos K. Theoretical Foundations of Polymer Processing. - M.: Chemistry, 1984. - 632 p.

16. Bernhardt E. Recycling of thermoplastic materials. M.: Chemistry, 1965. - 747p.

17. Silin V.A. Dynamics of plastic recycling processes in extrusion machines. - M.: Mechanical engineering, 1972. - $150 \mathrm{p}$.

18. V. Ivanova, D. Madrakhimov. Direct flow drawing equipment performance reliability and increase of its effectiveness. https://doi.org/10.1051/e3sconf/20191390105 7

19. $\bar{V}$. Tsipkia, V. Ivanova. Modeling of a resourcesaving method of drawing. https://doi.org/10.1051/e3sconf/201913901073 